\title{
Successes and failures of Bethe ansatz density functional theory
}

\author{
Stefan Schenk, Michael Dzierzawa, Peter Schwab, and Ulrich Eckern \\ Institut für Physik, Universität Augsburg, 86135 Augsburg, Germany \\ (Received 20 February 2008; revised manuscript received 11 August 2008; published 2 October 2008)
}

\begin{abstract}
The response of a one-dimensional fermion system is investigated using density functional theory (DFT) within the local-density approximation (LDA) and compared with exact results. It is shown that DFT-LDA reproduces surprisingly well some of the characteristic features of the Luttinger liquid, for example, the dispersion of the collective charge excitations. On the other hand, the approximation fails even qualitatively for details of the dynamic response and for quantities for which backscattering is important, i.e., those quantities which are crucial for an accurate description of transport. In particular, the Drude weight in the presence of a single impurity is discussed.
\end{abstract}

DOI: 10.1103/PhysRevB.78.165102

PACS number(s): 71.10.Pm, 71.15.Mb, 73.21.Hb

\section{INTRODUCTION}

Density functional theory (DFT) is the most efficient and powerful tool for determining the electronic structure of solids. While originally developed for continuum electron systems with Coulomb interaction, ${ }^{1,2}$ DFT has also been applied to lattice models, such as the Hubbard model. ${ }^{3-6}$ One goal of these studies was to develop new approaches to correlated electron systems: lattice models often allow for exact solutions - either analytically or based on numerics-which hence can serve as benchmarks for assessing the quality of approximations that usually have to be made when using DFT. Very popular in solid-state applications is the localdensity approximation (LDA) where the exchangecorrelation energy of the inhomogeneous system under consideration is constructed via a local approximation from the homogeneous electron system. Recently a lattice version of LDA has been suggested for one-dimensional systems ${ }^{6,7}$ where the underlying homogeneous system can be solved using the Bethe ansatz. For example, the Bethe ansatz LDA has been applied to study ultracold fermions in onedimensional optical lattices, ${ }^{8,9}$ Friedel oscillations in onedimensional metals, ${ }^{10}$ quantum spin chains, ${ }^{10}$ the Mott gap in the Hubbard model, ${ }^{7}$ and strongly correlated electrons out of equilibrium. $^{11}$

For small systems and not too strong interactions, LDA in most cases produces reasonable results - which can be obtained with much less numerical effort than needed when using more accurate methods, such as exact diagonalization or the density-matrix renormalization group. This led to the hope that the LDA may serve as a useful tool for large systems where the numerical effort for the more accurate methods is too expensive.

After recalling in Sec. II the theoretical background for the Bethe ansatz LDA, we will study in detail the LDA solution of spinless fermions in one dimension. In Sec. III we determine the charge susceptibility and discuss, in particular, questions of stability as well as the static and the dynamic response. In Sec. IV, we study the Drude weight (which can be related to the conductivity) in the presence of a single impurity, and finally, present our conclusions in Sec. V. From the size dependence of our results, we find that for large systems, LDA predictions are qualitatively incorrect even for weak interaction.

\section{FORMALISM}

We consider a one-dimensional model of spinless fermions described by the Hamiltonian

$$
\hat{H}=-t \sum_{i}\left(\hat{c}_{i}^{+} \hat{c}_{i+1}+\text { H.c. }\right)+V \sum_{i} \hat{n}_{i} \hat{n}_{i+1}+\sum_{i} v_{i} \hat{n}_{i},
$$

where $\hat{c}_{i}^{+}\left(\hat{c}_{i}\right)$ creates (annihilates) a fermion at site $i, t$ is the hopping parameter, $V$ is the nearest-neighbor interaction, and $v_{i}$ is an arbitrary local potential. The lattice consists of $L$ sites (the lattice constant $a$ is set to one), and periodic boundary conditions are assumed.

The lattice version ${ }^{3}$ of DFT relies on the fact that there is a one-to-one correspondence between the potentials $\left\{v_{i}\right\}$ and the ground-state expectation values of the site occupations $\left\{n_{i}\right\}$. Therefore it is-in principle-possible to express all quantities that can be obtained from the ground-state wave function as a function (or functional in the continuous case) of the densities. The site occupations as a function of the potentials can, of course, be found from derivatives of the ground-state energy with respect to the local potential,

$$
n_{i}=\frac{\partial E_{0}}{\partial v_{i}}
$$

On the other hand, in order to determine the potentials from the densities it is convenient to define the function

$$
F\left(\left\{n_{i}\right\}\right)=\min _{\Psi \rightarrow\left\{n_{i}\right\}}\langle\Psi|\hat{T}+\hat{V}| \Psi\rangle,
$$

where $\Psi \rightarrow\left\{n_{i}\right\}$ indicates that the minimization is constrained to such wave functions $\Psi$ that yield the given site occupation, i.e., $\left\langle\Psi\left|\hat{n}_{i}\right| \Psi\right\rangle=n_{i}$. Here $\hat{T}$ and $\hat{V}$ are the kinetic and interaction parts of the Hamiltonian (1), respectively. The ground-state energy is obtained by minimizing the function $E\left(\left\{n_{i}\right\}\right)=F\left(\left\{n_{i}\right\}\right)+\sum_{i} v_{i} n_{i}$ with respect to $n_{i}$. This yields the condition

$$
\frac{\partial F}{\partial n_{i}}+v_{i}=0
$$

which, of course, is purely formal unless $F$ or at least a reasonable approximation for it is available. 
A major step toward the practical implementation of DFT was the idea of Kohn and Sham ${ }^{2}$ to employ a noninteracting auxiliary Hamiltonian $\hat{H}^{s}$ in order to calculate the groundstate density profile. In the present case,

$$
\hat{H}^{s}=\hat{T}+\sum_{i} v_{i}^{s} \hat{n}_{i}
$$

where the potentials $v_{i}^{s}$ have to be chosen such that in the ground state of $\hat{H}^{s}$ the site occupations $n_{i}$ are the same as in the interacting model. Performing the same steps as before, one obtains the conditions

$$
\frac{\partial F^{s}}{\partial n_{i}}+v_{i}^{s}=0 .
$$

Combining Eqs. (4) and (6) yields

$$
v_{i}^{s}=v_{i}+\frac{\partial}{\partial n_{i}}\left(F-F^{s}\right)=: v_{i}+v_{i}^{\mathrm{H}}+v_{i}^{\mathrm{xc}},
$$

where $v_{i}^{\mathrm{H}}=V\left(n_{i+1}+n_{i-1}\right)$ is the Hartree potential, and $v_{i}^{\mathrm{xc}}$ is the so-called exchange-correlation potential. The minimization problem of DFT is thus mapped onto the diagonalization of the one-particle Hamiltonian $\hat{H}^{s}$ supplemented with the self-consistent condition (7). However, there remains the problem of finding a practical approximation for the exchange-correlation potential $v_{i}^{\mathrm{xc}}$. Most DFT studies of lattice models have so far relied on the LDA where the groundstate energy density $\epsilon_{i}$ of the inhomogeneous system is approximated by the energy density of a homogeneous system at the same density. In the present case this quantity can be calculated from the Bethe ansatz equations; ${ }^{12}$ hence

$$
\left[v_{i}^{\mathrm{xc}}\right]_{\mathrm{LDA}}=\frac{\partial}{\partial n_{i}}\left[\epsilon^{\mathrm{BA}}\left(n_{i}\right)-\epsilon^{\mathrm{H}}\left(n_{i}\right)\right],
$$

where $\epsilon^{\mathrm{BA}}(n)$ is the Bethe ansatz energy per site of a homogeneous system with particle density $n$ and $\epsilon^{\mathrm{H}}(n)$ is the corresponding energy density in the Hartree approximation.

\section{SUSCEPTIBILITY}

In order to assess the results based on LDA and to discuss their validity, it is appropriate to recall first the phase diagram of the model under consideration. ${ }^{12,13}$

In the homogeneous case and away from half filling, one finds for all values of the interaction parameter $V / t>-2$ a Luttinger liquid phase, i.e., there is no long-range charge order and the low-energy excitations are gapless collective charge excitations. For $V / t<-2$, phase separation is found. Finally, at half filling and for $V / t>2$ the model exhibits long-range charge order and a charge gap opens. ${ }^{14}$

Figure 1 shows the exchange-correlation potential $v^{\mathrm{xc}}(n)$ obtained from the Bethe ansatz [compare Eq. (8)] for several values of the interaction strength $V$. Due to particle-hole symmetry, we have $v^{\mathrm{xc}}(1-n)=-v^{\mathrm{xc}}(n)$. Furthermore, for $V / t>2$ there is a discontinuity at $n=1 / 2$ related to the opening of the charge gap.

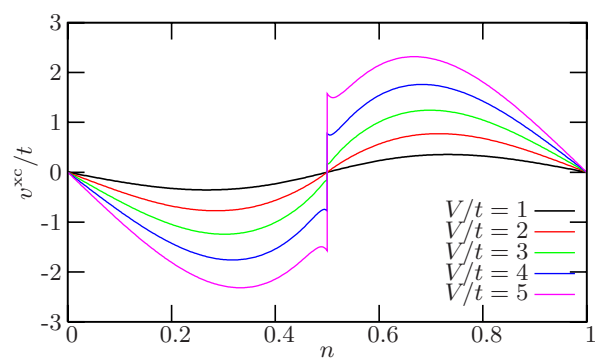

FIG. 1. (Color online) Exchange-correlation potential $v^{\mathrm{xc}}$ [within LDA as obtained from Eq. (8)] of the one-dimensional spinless fermion model as a function of the density $n$ for several values of the nearest-neighbor interaction $V$.

\section{A. Stability}

First we study the stability of the homogeneous LDA solution by considering the charge susceptibility $\chi(q)$. Generally, the susceptibility can be expressed as ${ }^{15,16}$

$$
\chi(q)=\frac{\chi_{0}(q)}{1+\left[V(q)+f^{\mathrm{xc}}(q)\right] \chi_{0}(q)},
$$

where $q$ is the wave vector, $f^{\mathrm{xc}}(q)$ is the Fourier transform of $f_{i-j}^{\mathrm{xc}}=\partial v_{i}^{\mathrm{xc}} / \partial n_{j}$, and $V(q)=2 V \cos q ; \chi_{0}(q)$ is the static susceptibility of the auxiliary system given by $(L \rightarrow \infty)$

$$
\chi_{0}(q)=\frac{1}{4 \pi t \sin (q / 2)} \ln \left|\frac{\sin (q / 2)+\sin k_{F}}{\sin (q / 2)-\sin k_{F}}\right|,
$$

where $k_{F}$ is the Fermi wave vector. The stability boundary of the homogeneous density profile is determined by the condition that the static susceptibility becomes infinite and changes sign; this happens whenever the denominator in Eq. (9) vanishes, i.e., for $V(q)+f^{\mathrm{xc}}(q)=-\chi_{0}^{-1}(q)$. Due to the logarithmic divergence of $\chi_{0}(q)$ for $q \rightarrow 2 k_{F}$, this is equivalent to the condition that $V\left(2 k_{F}\right)+f^{\mathrm{xc}}\left(2 k_{F}\right)$ changes sign. Notice that within LDA the function $f^{\mathrm{xc}}(q) \rightarrow f_{\mathrm{LDA}}^{\mathrm{xc}}$ is independent of $q$. Figure 2 shows the region of stability in the $n-V$ plane obtained within LDA both for the infinite system and for finite systems of length $L=100$ and 1000, respectively. For $L \rightarrow \infty$ only systems with density near $1 / 2$ and not too strong interaction are stable; further away from half filling, the homogeneous solution is unstable for arbitrarily weak interaction.

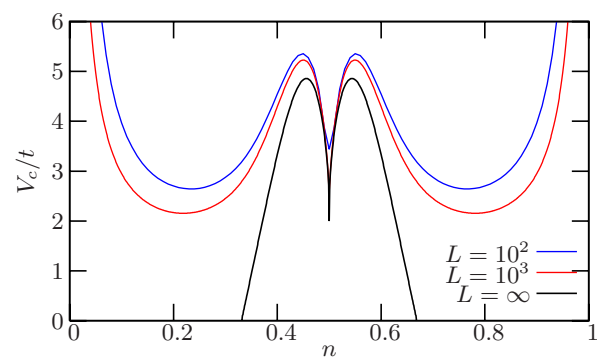

FIG. 2. (Color online) Critical interaction strength $V_{c}$ above which the LDA susceptibility is negative, indicating an instability of the system with respect to charge ordering. In the infinite system, the stable region is localized near half filling from $n_{c} \approx 0.331$ to 1 $-n_{c}$. For finite system size and weak interaction, LDA is stable for all densities. 


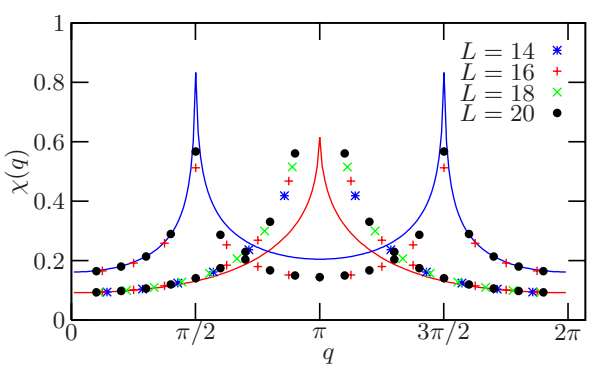

FIG. 3. (Color online) Static susceptibility $\chi(q)$ (in units of $t^{-1}$ ) in LDA for $V / t=1$ at half filling ( $L=202$; one-peak curve) and quarter filling ( $L=204$; two-peak curve). The symbols are results from exact diagonalization for systems of up to $L=20$ sites.

For an accurate determination of the phase boundary we investigate the weak-interaction case in more detail. We find

$$
f_{\text {LDA }}^{\mathrm{xc}}=\frac{\partial^{2}}{\partial n^{2}}\left(\epsilon^{\mathrm{BA}}-\epsilon^{\mathrm{H}}\right)=-V\left(2 k_{F}\right)+\mathcal{O}\left(V^{2}\right)
$$

so that in the first order in the interaction no conclusion about the stability can be drawn: the second-order correction to the ground-state energy $\epsilon_{2}$ is needed. Numerically we find that its second derivative with respect to the density $\epsilon_{2}^{\prime \prime}(n)$ changes sign at $n_{c} \approx 0.331$ thus limiting the range of stability to $n_{c}<n<1-n_{c}$ at weak coupling. This result should be contrasted with the Hartree approximation $\left(f^{\mathrm{xc}}=0\right)$ where the homogeneous system is stable only for $V\left(2 k_{F}\right)>0$, i.e., below quarter and above three quarter filling, and with the exact ground state where a charge instability of the homogeneous system occurs only at half filling for $V>2 t$.

There are, however, very pronounced finite-size effects that strongly enlarge the actual region of stability within LDA. Since $\chi_{0}\left(2 k_{F}\right)$ diverges only logarithmically with system size $L$, the critical interaction strength approaches zero very slowly, $V_{c}(L) \sim 1 / \sqrt{\ln L}$. As a consequence, for finite systems and from weak to intermediate interaction strength, the homogeneous LDA solution is stable for all densities, as can be seen in Fig. 2 for $L=100$ and 1000.

\section{B. Static response}

Here we investigate the static susceptibility, i.e., its $q$ dependence, in more detail. In Fig. 3 we show $\chi^{\operatorname{LDA}}(q)$ for $V / t=1$ in comparison with the exact susceptibility obtained from numerical diagonalization of small systems. As to be expected, in the long-wavelength limit $q \rightarrow 0$, perfect agreement is found. Technically, there is a cancellation between the susceptibility $\chi_{0}^{-1}(0)=2 \pi t \sin k_{F}$ and the second derivative of the Hartree energy $\epsilon^{\mathrm{H}}=-(2 t / \pi) \sin k_{F}+V n^{2}$ with respect to $n=k_{F} / \pi$. Therefore,

$$
\chi^{\mathrm{LDA}}(q \rightarrow 0)=\left(\frac{\partial^{2} \epsilon^{\mathrm{BA}}}{\partial n^{2}}\right)^{-1}=\frac{1}{L} \frac{\partial N}{\partial \mu},
$$

which is the exact uniform susceptibility of the interacting system. Unfortunately, already the next to leading contribution $\sim q^{2}$ is not obtained correctly within LDA. At half filling the discrepancy between the LDA susceptibility and the exact one becomes more and more pronounced for $q \rightarrow 2 k_{F}$

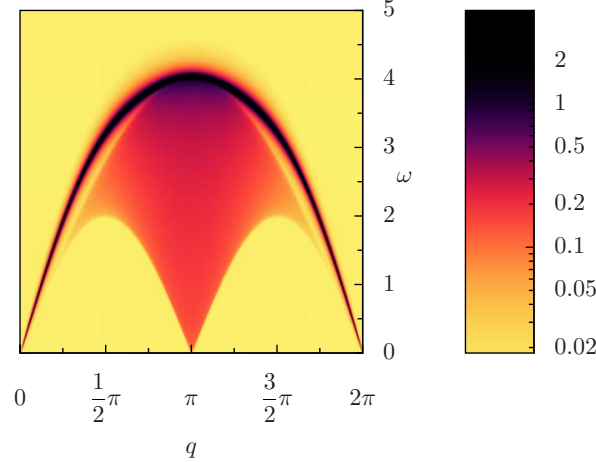

FIG. 4. (Color online) Imaginary part of the dynamical susceptibility $\chi^{\operatorname{ALDA}}(q, \omega)$ (in units of $\left.t^{-1}\right)$.

$=\pi$. At $q=2 k_{F}$ the exact susceptibility increases with the system size (not shown in the figure) and diverges with a power law, while in LDA there is only a cusp. The cusp value itself remains finite and approaches $\chi^{\mathrm{LDA}}(\pi)=1.668 / t$ for $L \rightarrow \infty$.

At quarter filling $\chi^{\mathrm{LDA}}(q)$ is very close to the exact susceptibility for $q<2 k_{F}$, while for $q>2 k_{F}$ there is a clear discrepancy. For $q=2 k_{F}=\pi / 2$ the exact result again is strongly size dependent and diverges for $L \rightarrow \infty$, while within LDA the susceptibility diverges already at a finite system size since at quarter filling one is already outside the range of stability of LDA.

\section{Dynamic response}

DFT as presented in Sec. II is a ground-state theory. However, a time-dependent generalization ${ }^{17}$ of DFT is available, which allows one to calculate frequency-dependent response functions. ${ }^{15}$ The dynamic susceptibility of the homogeneous system is of the same form as Eq. (9) with the only differences that $\chi_{0}(q)$ has to be replaced by $\chi_{0}(q, \omega)$ and $f^{\mathrm{xc}}(q)$ by $f^{\mathrm{xc}}(q, \omega)$. In a simple approximation called adiabatic localdensity approximation ${ }^{18}$ (ALDA), one neglects this frequency dependence and uses the function $f^{\mathrm{xc}}$ already known from LDA $f^{\mathrm{xc}}(q, \omega) \rightarrow f_{\mathrm{LDA}}^{\mathrm{xc}}$. The corresponding approximation for the susceptibility is denoted as $\chi^{\mathrm{ALDA}}(q, \omega)$.

Figure 4 shows the imaginary part of $\chi^{\operatorname{ALDA}}(q, \omega)$ in the $q-\omega$ plane for a half-filled system and $V / t=1$. A continuum of excitations in the frequency range $2 t|\sin q|<\omega$ $<4 t \sin (q / 2)$ is apparent, which can be identified with the particle-hole continuum. Note that the spectral weight of the particle-hole excitations vanishes for $q \rightarrow 0$ as expected for a Luttinger liquid.

Above this continuum we find a well-defined branch of collective excitations with linear dispersion for small $q$. Analytically, the dispersion of the collective excitations can be obtained from the singularities of the susceptibility, i.e., by considering the zeros of the denominator of $\chi(q, \omega)$. In the low frequency and small wave-vector limit, the susceptibility agrees with the Luttinger liquid result

$$
\chi(q, \omega) \approx \frac{1}{L} \frac{\partial N}{\partial \mu} \frac{(q v)^{2}}{(q v)^{2}-\omega^{2}},
$$

where $v$ is the velocity of the collective excitations. Within the adiabatic local-density approximation, the velocity is given by 


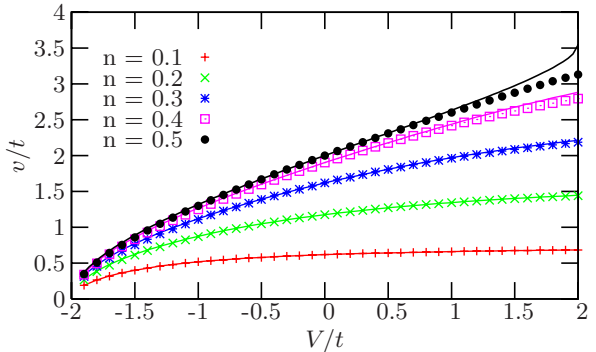

FIG. 5. (Color online) Charge velocity $v$ as a function of the interaction strength $V$ for densities $n=0.1,0.2,0.3,0.4$, and 0.5 (from bottom to top). Exact values from Bethe ansatz (symbols) in comparison with the results obtained within ALDA (solid lines) from Eq. (14).

$$
v_{\mathrm{ALDA}}=v_{F} \sqrt{1+\frac{2 V+f_{\mathrm{LDA}}^{\mathrm{xc}}}{\pi v_{F}}},
$$

where $v_{F}=2 t \sin k_{F}$ is the Fermi velocity. To linear order in the interaction, $v_{\text {ALDA }}$ agrees with the exact result. In Fig. 5 we compare $v_{\text {ALDA }}$ with the exact value obtained from the Bethe ansatz for various interaction strengths and densities. For small densities, there is nearly perfect agreement between the two values. The largest deviation occurs at half filling at the critical point $V=2 t$ where the error is $2 / \sqrt{\pi}-1$, which is about $13 \%$. We want to emphasize that within the random-phase approximation, i.e., neglecting the ALDA correction factor $f^{\mathrm{xc}}$ in Eq. (14), one never obtains the correct charge velocity except for $V=0$. From the discrepancy between $v_{\text {ALDA }}$ and the exact value, one concludes that the frequency and wave-vector-dependent function $f^{\mathrm{xc}}(q, \omega)$ is nonanalytic in the $q, \omega \rightarrow 0$ limit.

Recently several exact results for the dynamic response beyond the free-boson description of the Luttinger liquid have been obtained; ${ }^{19-22}$ as to be expected, these results are not reproduced within ALDA. For example, the structure factor (see, in particular, Fig. 1 of Ref. 20 and Fig. 1 of Ref. 21) considered as a function of $\omega$ for fixed $q$ displays a continuum of states in a finite range $\omega_{-}<\omega<\omega_{+}$where $\omega_{ \pm}$ are $q$ and interaction dependent. In addition, for a repulsive interaction, a power-law divergence near $\omega_{-}$is found, implying a shift of spectral weight toward the lower end of the continuum. In contrast, ALDA incorrectly shows a welldefined collective mode for finite $q$ clearly separated from (and above) the continuum, as discussed above; see Fig. 4.

\section{SINGLE IMPURITY}

Finally, we consider the spinless fermion model for a nonzero potential $v_{i}$. As an important example in this context, we consider the case of a single impurity, i.e., $v_{i}=v_{\text {imp }}$ at the impurity site and $v_{i}=0$ elsewhere. It is well known that in one-dimensional interacting systems the reflection and transmission probabilities for scattering at an impurity are strongly renormalized. ${ }^{23-25}$ Here we address the question of whether this renormalization is captured by the LDA-with negative conclusion.

Let us first consider the simple picture for the origin of the renormalization that was developed by Matveev et al. ${ }^{25}$

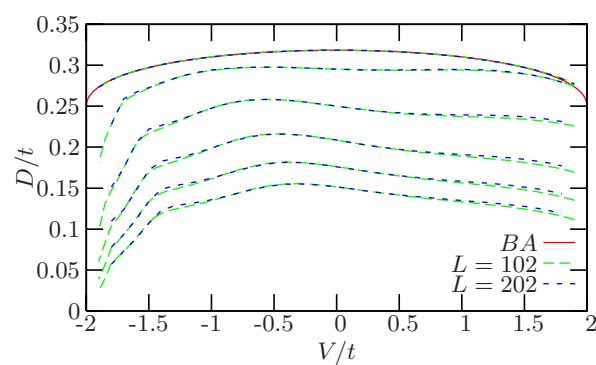

FIG. 6. (Color online) Drude weight $D$ for a half-filled system with a single impurity as function of the interaction strength $V$ for several values of the impurity strength $v_{\text {imp }} / t=0,1,2,3,4$, and 5 (from top to bottom). The solid curve is the Bethe ansatz result for the clean system; the long- and short-dashed curves are LDA results for $L=102$ and $L=202$, respectively.

Around an impurity, the density is disturbed, and in the presence of electron-electron interaction this modulation (Friedel oscillations) leads to additional scattering. In particular, the Friedel oscillations are the origin of enhanced backscattering. To linear order in the interaction, the correction to the transmission probability for a wave vector $q$ close to $k_{F}$ is given by ${ }^{25}$

$$
\delta \mathcal{T}=-2 \alpha \mathcal{T}_{0}\left(1-\mathcal{T}_{0}\right) \ln \left(\frac{1}{\left|q-k_{F}\right|}\right),
$$

where $\mathcal{T}_{0}$ is the bare value; the dimensionless parameter $\alpha$ characterizes the interaction strength. It is given by the sum of a Hartree and an exchange contribution $\alpha=\alpha_{H}+\alpha_{x}$ with $\alpha_{H}=-V\left(2 k_{F}\right) / 2 \pi v_{F}$ and $\alpha_{x}=V(0) / 2 \pi v_{F}$. By summation of the leading divergences to all orders in the interaction using a renormalization-group approach, it is found that even for a weak defect the transmission approaches zero as $q \rightarrow k_{F}$ (repulsive interaction). Repeating the arguments leading to Eq. (15) within DFT and for a weak impurity, we find $\alpha \rightarrow$ $-\left[V\left(2 k_{F}\right)+f^{\mathrm{xc}}\left(2 k_{F}\right)\right] / 2 \pi v_{F}$. Since $f_{\mathrm{LDA}}^{\mathrm{xc}}=-V\left(2 k_{F}\right)$, this singular correction to the transmission is zero; i.e., $\mathcal{T}$ is not renormalized in DFT-LDA.

To substantiate this finding numerically, we calculate the Drude weight for the single-impurity case. The Drude weight $D$ is defined as the response of the system to a change of boundary conditions according to

$$
D=\left.\frac{L}{2} \frac{d^{2} E}{d \varphi^{2}}\right|_{\varphi=0},
$$

where $E(\varphi)$ is the ground-state energy. The parameter $\varphi$ characterizes the twist in the boundary conditions: $\varphi=0$ corresponds to periodic and $\varphi=\pi$ to antiperiodic boundary conditions. ${ }^{23,26}$ The Drude weight is closely related to the transmission through the defect, and in the noninteracting system-where $\mathcal{T}$ is not renormalized-the size dependence of $D$ is negligible. In the interacting system, on the other hand, the transmission coefficient for $\left(q-k_{F}\right) \approx v_{F} / L$ is relevant as discussed above. Correspondingly, the Drude weight increases (decreases) algebraically with system size for repulsive (attractive) interaction. ${ }^{23-25}$

In Fig. 6 we present our LDA results for the Drude weight 
at half filling for different system sizes $(L=102$ and $L=202)$ and different values of the impurity strength. Unlike the (numerically) exact results, ${ }^{23}$ we do not observe any dependence on system size within the LDA in agreement with the perturbative argument given in relation with Eq. (15).

\section{SUMMARY}

We studied in detail the Bethe ansatz LDA for spinless fermions in one dimension. The numerical effort of the method is comparable to the Hartree (or Hartree-Fock) approximation. As a major improvement with respect to the Hartree approximation, the Bethe ansatz LDA correctly predicts a noncharge-ordered ground state for a large range of parameters. Both the static and the dynamic density response functions agree reasonably well with the exact results. In particular, for low density and $q<2 k_{F}$, the static susceptibility obtained within LDA is almost indistinguishable from the exact one. In the dynamic case a very good agreement for the velocity of collective charge excitations is found. On the other hand, the adiabatic LDA fails to describe details of the dynamics of the spinless fermion model, namely, those that are related to aspects beyond the Luttinger model; see Sec. III C.

Furthermore, the LDA does not capture the fact that the system is critical with respect to a charge-ordering phase transition. Whereas the exact susceptibility has a power-law singularity at $q=2 k_{F}$, the LDA susceptibility remains either finite or the system develops charge ordering for very large system size. As a consequence physical quantities that are related to the $2 k_{F}$-periodic charge oscillations are described incorrectly within the local-density approximation. Examples are the Friedel oscillations around a defect, the interaction renormalization of the reflection and transmission probability (and therefore the conductance), and the Drude weight.

We emphasize that our focus has been on a "simple" interacting-fermion model in one dimension utilizing exact results available for the model (for the homogeneous case). The situation is entirely different from "standard" applications of DFT-LDA in solid-state physics, in which case the ionic and electronic charge densities vary strongly. Thus, in solid-state physics (and in chemistry), there has been extensive research on improving the exchange-correlation potential, for example, within the generalized gradient approximation, ${ }^{27}$ i.e., by including derivatives of the density, or within the LDA $+U$ approach, ${ }^{28}$ which captures aspects of the (local) Coulomb interaction between localized orbitals.

It remains to be seen whether any of these concepts are useful for the one-dimensional models studied here. From our results, it appears to be promising to pursue the exact exchange or optimized effective potential approach ${ }^{29}$ further; however, it is at present not clear whether the "critical" $2 k_{F}$ scattering in one dimension can be captured properly. Nevertheless we are confident that the Bethe ansatz LDA, combined with numerically determined exact exchangecorrelation potentials, ${ }^{30}$ is a very useful approach for relatively short inhomogeneous systems.

\section{ACKNOWLEDGMENTS}

Helpful discussions with Peter Schmitteckert and Cosima Schuster are gratefully acknowledged. This work was supported by the Deutsche Forschungsgemeinschaft through Collaborative Research Center (SFB) 484.
${ }^{1}$ P. Hohenberg and W. Kohn, Phys. Rev. 136, B864 (1964).

${ }^{2}$ W. Kohn and L. J. Sham, Phys. Rev. 140, A1133 (1965).

${ }^{3}$ O. Gunnarsson and K. Schönhammer, Phys. Rev. Lett. 56, 1968 (1986).

${ }^{4}$ K. Schönhammer and O. Gunnarsson, J. Phys. C 20, 3675 (1987).

${ }^{5}$ K. Schönhammer, O. Gunnarsson, and R. M. Noack, Phys. Rev. B 52, 2504 (1995).

${ }^{6}$ N. A. Lima, M. F. Silva, L. N. Oliveira, and K. Capelle, Phys. Rev. Lett. 90, 146402 (2003).

${ }^{7}$ N. A. Lima, L. N. Oliveira, and K. Capelle, Europhys. Lett. 60, 601 (2002).

${ }^{8}$ Gao Xianlong, M. Polini, M. P. Tosi, V. L. Campo, Jr., K. Capelle, and M. Rigol, Phys. Rev. B 73, 165120 (2006).

${ }^{9}$ Gao Xianlong, M. Rizzi, M. Polini, R. Fazio, M. P. Tosi, V. L. Campo, Jr., and K. Capelle, Phys. Rev. Lett. 98, 030404 (2007).

${ }^{10}$ F. C. Alcaraz and K. Capelle, Phys. Rev. B 76, 035109 (2007).

${ }^{11}$ C. Verdozzi, arXiv:0707.2317 (unpublished).

${ }^{12}$ C. N. Yang and C. P. Yang, Phys. Rev. 150, 321 (1966). The spin-1/2 chain studied in Ref. 12, also known as the XXZ model, can be mapped to the fermionic Hamiltonian (1) applying a Jordan-Wigner transformation; see, for example, H. Fukuyama and H. Takayama, in Electronic Properties of Inorganic QuasiOne-Dimensional Compounds, edited by P. Monceau (Reidel,
Dordrecht, 1985), Pt. I, pp. 41-104. For a recent review on magnetism in one dimension, see H.-J. Mikeska and A. K. Kolezhuk, in Quantum Magnetism, Lecture Notes in Physics Vol. 645, edited by U. Schollwöck, J. Richter, D. J. J. Farnell, and R. F. Bishop (Springer, Berlin, 2004), pp. 1-83.

${ }^{13}$ F. D. M. Haldane, Phys. Rev. Lett. 45, 1358 (1980). In this paper, the term "Luttinger liquid" was introduced.

${ }^{14}$ Phase separation corresponds to a ferromagnetic phase, and long-range charge order (at half filling) to an antiferromagnetic phase (at zero magnetic field) in the spin model, respectively.

${ }^{15}$ E. K. U. Gross and W. Kohn, Phys. Rev. Lett. 55, 2850 (1985). Note that our Eq. (9) reduces to the standard RPA form for $f^{\mathrm{xc}}(q) \equiv 0$.

${ }^{16}$ We define $\chi$ and $\chi_{0}$ with a different sign compared to Ref. 15 . With our choice, which is the standard one in nonequilibrium statistical physics, the static susceptibility is positive.

${ }^{17}$ E. Runge and E. K. U. Gross, Phys. Rev. Lett. 52, 997 (1984).

${ }^{18}$ A. Zangwill and P. Soven, Phys. Rev. Lett. 45, 204 (1980).

${ }^{19}$ R. G. Pereira, J. Sirker, J.-S. Caux, R. Hagemans, J. M. Maillet, S. R. White, and I. Affleck, Phys. Rev. Lett. 96, 257202 (2006).

${ }^{20}$ M. Pustilnik, M. Khodas, A. Kamenev, and L. I. Glazman, Phys. Rev. Lett. 96, 196405 (2006).

${ }^{21}$ R. G. Pereira, S. R. White, and I. Affleck, Phys. Rev. Lett. 100, 027206 (2008). 
${ }^{22}$ V. V. Cheianov and M. Pustilnik, Phys. Rev. Lett. 100, 126403 (2008).

${ }^{23}$ P. Schmitteckert, T. Schulze, C. Schuster, P. Schwab, and U. Eckern, Phys. Rev. Lett. 80, 560 (1998).

${ }^{24}$ C. L. Kane and M. P. A. Fisher, Phys. Rev. Lett. 68, 1220 (1992).

${ }^{25}$ K. A. Matveev, D. Yue, and L. I. Glazman, Phys. Rev. Lett. 71, 3351 (1993).

${ }^{26}$ For a system of charged particles on a ring in a perpendicular magnetic field, $\varphi=2 \pi \phi / \phi_{0}$, where $\phi$ denotes the magnetic flux and $\phi_{0}$ the flux quantum. See, for example, U. Eckern and P.
Schwab, Adv. Phys. 44, 387 (1995), and references therein.

${ }^{27}$ D. C. Langreth and J. P. Perdew, Phys. Rev. B 21, 5469 (1980).

${ }^{28}$ V. I. Anisimov, J. Zaanen, and O. K. Andersen, Phys. Rev. B 44, 943 (1991); A. I. Liechtenstein, V. I. Anisimov, and J. Zaanen, ibid. 52, R5467 (1995).

${ }^{29}$ See, for example: T. Grabo and E. K. U. Gross, Chem. Phys. Lett. 240, 141 (1995); V. N. Staroverov, G. E. Scuseria, and E. R. Davidson, J. Chem. Phys. 125, 081104 (2006), and references therein.

${ }^{30}$ P. Schmitteckert and F. Evers, Phys. Rev. Lett. 100, 086401 (2008). 